\title{
Revisión de la sección Calceolaria (Calceolariaceae) en Chile
}

\section{Revision of section Calceolaria (Calceolariaceae) in Chile}

\author{
Pamela Puppo ${ }^{1} \&$ Patricio Novoa ${ }^{2}$ \\ ${ }^{1}$ Centro de Investigação em Biodiversidade e Recursos Genéticos (CIBIO), Universidade do Porto -Campus Vairão, R. Monte- \\ Crasto, 4485-661 Vairão, Portugal. \\ 2Jardín Botánico Nacional, Camino el Olivar 305, El Salto, Viña del Mar, Chile. \\ p.puppo@cibio.up.pt; p_puppo@hotmail.com
}

\begin{abstract}
RESUMEN
Calceolaria se caracteriza por sus flores generalmente amarillas, bilabiadas con el labio inferior sacciforme y dos estambres. La sección Calceolaria contiene alrededor de 10 especies, todas herbáceas e higrófilas con una morfología estaminal particular. Las tecas son dimorfas y el conectivo alargado en diversas formas. En Chile sólo dos especies de esta sección, C. pinnata y C. tripartita, fueron reportadas en las diversas publicaciones hechas sobre la flora de este país desde el siglo XVIII. Es más, el uso de nombres inválidos como C. scabiosifolia Roem. \& Schult. contribuyeron a la mala identificación de los ejemplares de esta sección confundiendo aún más su taxonomía. El objetivo del presente artículo es hacer la primera revisión de las especies de esta sección presentes en Chile, así como una revisión completa de la literatura que hace referencia a las mismas. Se incluye una clave para su identificación, descripciones de cada una, ilustraciones, comentarios sobre su distribución y la evaluación de su estado de conservación en el país según la metodología de la UICN.
\end{abstract}

Palabras Clave: Scrophulariaceae, calceolarias de agua, capachitos, UICN.

\begin{abstract}
Calceolaria is characterized by its bilabiate generally yellow flowers with the lower lip saccate and two stamens. Section Calceolaria has near 10 species all herbaceous and hygrophilous with a particular staminal morphology. The thecae are dimorphic and the connective is elongated in various ways. In Chile, only two species of this section, $C$. pinnata and $C$. tripartita, were known from the different flora publications since the $18^{\text {th }}$ century. Moreover, the use of invalid species names such as C. scabiosifolia Roem. \& Schult. contributed to the misidentification of the material of this section, further complicating its taxonomy. The aim of the present work is to make the first revision of the species of this section present in Chile as well as presenting a thorough revision of the literature that makes reference to them. A key for the species identification is included as well as descriptions, illustrations, comments on their distribution, and their conservation status in the country according to the IUCN methodology.
\end{abstract}

KEYwords: Scrophulariaceae, water calceolarias, capachitos, IUCN.

\section{INTRODUCCIÓN}

El género Calceolaria L. está constituido por aproximadamente 250 especies distribuidas desde México hasta el sur de Argentina (Molau 1988). Se caracteriza por su corola bilabiada con el labio inferior sacciforme provisto de una glándula secretora de aceite conocida como elaióforo (Vogel 1974). La corola es usualmente amarilla y el androceo está compuesto por dos estambres. Una única especie, $C$. triandra (Cav.) Vahl, presenta una corola trilobada y tres estambres. Esta especie pertenecía al género monotípico Porodittia G. Don hasta que análisis moleculares recientes probaron su pertenencia al género Calceolaria (Andersson 2006, Cosacov et al. 2009).

Tradicionalmente considerado como parte de la familia Scrophulariaceae, el género Calceolaria fue elevado al rango de familia cuando las Scrophulariaceae fueron encontradas polifiléticas (Olmstead \& Reeves 1995, Olmstead et al. 2001). Desde entonces, forma la familia Calceolariaceae junto con el género Jovellana Ruiz \& Pav.

La última revisión del género hecha como parte de las monografías de Flora Neotrópica (Molau 1988) subdividió el género en 3 subgéneros y cuantiosas secciones. Sin embargo, la mayoría de éstas fueron encontradas polifiléticas en 
análisis moleculares posteriores (Andersson 2006, Cosacov et al. 2009).

La sección Calceolaria es una de las pocas secciones monofiléticas del género Calceolaria. Está compuesta por cerca de 10 especies que se caracterizan por su hábito herbáceo, higrófilo, y tecas dimorfas con el conectivo diversamente alargado. En la revisión de Molau (1988), éste cita un único ejemplar de C. pinnata L. subsp. pinnata como presencia de esta sección en Chile. Más adelante, Ehrhart (2000) en su monografía del género para Chile reconoce esta especie como la única de la sección en el país citando otra colección además de la ya citada por Molau (1988). Revisando publicaciones más antiguas (ver Tabla I) encontramos que Ruiz \& Pavón (1798) no citan ninguna especie de esta sección para Chile. Gay (1845) y Reiche (1911) citan otra especie, C. scabiosifolia Sims además de C. pinnata. Reiche (1911 v.6, p.55) hace la observación de que una tercera especie, C. chelidonioides Kunth, "se halló en Chile, observada por Poeppig; pero como este viajero coleccionó también en otras partes de Sudamérica, esta indicación tal vez es errónea; en los últimos 80 años no se ha visto por nadie en Chile". Johow en 1948 describe la flora de Zapallar e incluye únicamente C. scabiosifolia. En 1985, Marticorena \& Quezada reportan además de C. pinnata y C. scabiosifolia, C. tripartita Ruiz \& Pav. y Villagrán et al. (2007) al hacer la reedición de la flora de Zapallar de Johow incluyen C. scabiosifolia como sinónimo de $C$. tripartita. Cabe recalcar que ninguno de estos autores (Gay 1845, Reiche 1911, Johow 1948, Marticorena \& Quezada 1985, Villagrán et al. 2007) cita ejemplares revisados. La lista de estas publicaciones así como las especies de la sección Calceolaria que citan para Chile ha sido incluida en la Tabla I.

Tabla I. Publicaciones conocidas de la Flora Chilena indicando las especies de la sección Calceolaria citadas por cada una. *Nótese que C. scabiosifolia Sims es sinónimo de C. chelidonioides. **Indica que la especie está presente en Chile, pero no cita ningún ejemplar revisado.

TABLE I. Known publications of the Flora of Chile indicating the species of section Calceolaria cited in each. *Note that C. scabiosifolia Sims is synonym to $C$. chelidonioides. **Indicates that the species is present in Chile but does not cite any revised collection.

\begin{tabular}{|c|c|c|c|c|}
\hline Publicaciones & $\begin{array}{l}\text { C. pinnata subsp. } \\
\text { pinnata }\end{array}$ & C. tripartita & C. chelidonioides & C. scabiosifolia Sims* \\
\hline \multicolumn{5}{|l|}{ Ruiz \& Pavón (1798) } \\
\hline Gay (1845) & & & & $\mathrm{x}$ \\
\hline Reiche (1911) & $\mathrm{x}$ & & & $\mathrm{x}$ \\
\hline Johow (1948) & & & & $\mathrm{x}$ \\
\hline $\begin{array}{l}\text { Marticorena \& Quezada } \\
\text { (1985) }\end{array}$ & $\mathrm{x}$ & $\mathrm{x}$ & & \\
\hline Molau (1988) & $\mathrm{x}$ & $* *$ & $* *$ & \\
\hline Ehrhart (2000) & $\mathrm{x}$ & $* *$ & & \\
\hline Villagrán et al. (2007) & & $\mathrm{x}$ & & \\
\hline $\begin{array}{l}\text { Puppo \& Novoa (El presente } \\
\text { artículo) }\end{array}$ & $\mathrm{x}$ & $\mathrm{x}$ & $\mathrm{x}$ & \\
\hline
\end{tabular}

Debido a esta confusión de nombres y a la falta de una revisión detallada de las especies de esta sección en Chile, la mayoría de las calceolarias de agua recolectadas en este país han sido determinadas como C. pinnata, C. scabiosifolia o C. tripartita. El presente artículo pretende actualizar el conocimiento de estas especies y otras de la sección Calceolaria que puedan estar presentes en Chile. Se incluyen descripciones de las especies encontradas, ilustraciones, distribución conocida, una clave para su identificación y finalmente se discute el estado de conservación de estas especies en Chile.

\section{MATERIALES Y MÉTODOS}

El material revisado para el presente artículo se encuentra depositado en los siguientes herbarios: CONC, F, G, MO, NY, PH, SGO, US. Los acrónimos de los herbarios usados en el texto están de acuerdo con el Index Herbariorum (Thiers 2011). La determinación del material fue realizada siguiendo la última revisión del género (Molau 1988). El material depositado en CONC y SGO fue examinado mediante fotografía de alta resolución. Las nuevas determinaciones hechas para el presente artículo han sido enviadas a los respectivos herbarios. Las descripciones de 
las especies reflejan la variación general de las especies y no sólo la encontrada en los especímenes chilenos. Para esto hemos hecho uso de las últimas dos revisiones del género (Molau 1988, Ehrhart 2000) así como revisiones hechas por Puppo en 2005, 2006 y 2008. Las ilustraciones de las especies fueron tomadas de Puppo $(2005,2008)$. El estado de conservación de las especies encontradas fue evaluado siguiendo las categorías y criterios de la UICN (2001).

\section{RESULTADOS}

En Chile se encuentran tres especies de la sección Calceolaria: C. chelidonioides, C. pinnata subsp. pinnata y C. tripartita.

Clave de identificación Para las especies de la Sección CaLCEOLARIA

1. Hierbas de hasta $60 \mathrm{~cm}$ alto; hojas pinnatisectas, pinnas profundamente lobadas; ambas tecas de los estambres fértiles ...... 2. C. pinnata subsp. pinnata

1'. Hierbas de hasta $2 \mathrm{~m}$ alto; hojas pinnatisectas, pinnas crenadas o serradas; teca anterior de los estambres estéril .........2 2. Hierbas de hasta $2 \mathrm{~m}$ alto; pinnas truncadas en la base, márgenes frecuentemente serrados o biserrados, peciolos frecuentemente ensanchados en la base y conspicuamente connados al tallo; teca posterior hasta 1,7 mm long., conectivo prolongado sin protuberancia dorsal (Fig. 1G) 1. C. chelidonioides

2'. Hierbas de hasta $1 \mathrm{~m}$ alto; pinnas ovadas, márgenes frecuentemente crenados, peciolos ligeramente ensanchados en la base no siempre connados al tallo; teca posterior hasta $2,5 \mathrm{~mm}$ de long., conectivo prolongado con una protuberancia dorsal (Fig. 3G)

3. C. tripartita

1. Calceolaria chelidonioides Kunth. Nov. Gen. Sp. Pl. 2: 378. 1818. Fagelia chelidonioides (Kunth.) Kuntze, Revis. Gen. Pl. 2: 459. 1891. TIPO: Ecuador. Pichincha: Cerro Javirac (=Panecillo), Quito, ca. $2750 \mathrm{~m}$. V-1802. Bonpland s.n. (lectotipo B-WILDD; isotipos F! [fragmento], G-DC, HAL, P). Fig. 1.

Calceolaria scabiosaefolia Sims., Bot. Mag. 50: t. 2405. 1823. TIPO: ilustración basada en material propagado de semillas chilenas por John Walker.

C. glutinosa Heer \& Regel, in Schlechtendal, Linnaea 24: 196. 1851. TIPO: Suiza. Cultivado en Zürich, de semillas colectadas por Warscewicz en Guatemala, herborizado en VIII-1848. Regel s.n. (lectotipo ZT).

Fagelia diversifolia Pennell, Addisonia 4: 73. 1919. TIPO: Colombia. Cundinamarca: Chipaque, SE de Bogotá, laderas E de la Cordillera Oriental, ca. 2650 m. 23-VIII-1917. Pennell 1320 (holotipo NY; isotipos US).

F. pinnatisecta Pennell, Proc. Acad. Nat. Sci. Philadelphia 72: 185. 1920. TIPO: Colombia. Caguetá: Hda. Balsillas en el Río Balsillas, E de Neiva, laderas E de la Cordillera Oriental, 2000-2100 m. 3-6-VIII-1917. Rusby \& Pennell 721 (holotipo NY; isotipos GH, US).

C. gracilis Kunth var. stenoptera Diels, Biblioth. Bot. 116: 140. 1937. TIPO: Ecuador. Pichincha: Cordillera Occidental, valle del Río Saloya, entre San Juan y Chiriboga, $2600 \mathrm{~m}$. 05-IX-1933. Diels 848 (holotipo B, destruido).

C. obscura Pennell, Proc. Acad. Nat. Sci. Philadelphia 97: 176. 1945. TIPO: Perú. Cuzco: Prov. Paucartambo, cerca al Río Yanamayo, debajo de Pillahuata, 2000-2200 m. 4-5-V1925. Pennell 14043 (holotipo PH; isotipos CUZ, F, GH, K, NY, US).

C. lilloi Descole \& Borsini, Lilloa 27: 75. 1953. TIPO: Argentina. Jujuy: Dept. Capital, a lo largo del camino a Yala. 12-I-1947. Descole \& Borsini 3544 (holotipo LIL).

C. cantensis López Guillén, Raymondiana 1:37. 1968. TIPO: Perú. Lima: Prov. Canta, a lo largo del Rio Chillón cerca a Canta. II-1967. López Guillén 3306 (holotipo SMF).

C. conocarpa Pennell subsp. irregularis López Guillén, Raymondiana 1: 71. 1968. TIPO: Perú. Sine loco, López Guillén 783 (holotipo SMF).

C. grandepinnata Edwin, Phytologia 19: 380. 1970. TIPO: Perú. Piura: Prov. Huancabamba, debajo de Canchaque, 1000 m. 21-23-III-1948. Pennell \& Ferreyra 14981 (holotipo $\mathrm{US}$; isotipos $\mathrm{BM}, \mathrm{GH}, \mathrm{NY}, \mathrm{PH})$.

Hierba de hasta $2 \mathrm{~m}$ de alto, tallos superiores glandularvillosos, verde-violáceos. Hojas pinnatisectas $(1,2) 6-$ $18 \times(0,4) 4-15 \mathrm{~cm}$, hasta 7 pares de pinnas laterales, 
pinnas agudas al ápice, truncadas en la base, márgenes frecuentemente serrados o aserrados, en especímenes pequeños o poco desarrollados las hojas pueden parecer enteras a pinnatipartidas; peciolos $(0,2) 2-8 \mathrm{~cm}$ long. frecuentemente con un conspicuo ensanchamiento en la base de hasta $6 \mathrm{~mm}$ y connados al tallo. Sépalos ovados, 2-6,5 x 1-5 mm, comúnmente verde-violáceos, agudos, margen entero o dentado, externamente glandular-pilosos. Corola 4-17 mm long., amarilla, glabra o con pequeñas glándulas. Estambres con una sola teca fértil (la posterior) de 0,6-1,7 mm de long., la teca anterior abortada, conectivo alargado 0,6-2,5 $\mathrm{mm}$ long., recto, liso, sin protuberancia dorsal. Cápsula cónica u ovada 5-10 mm long., glandular pubescente sobre todo en la base.
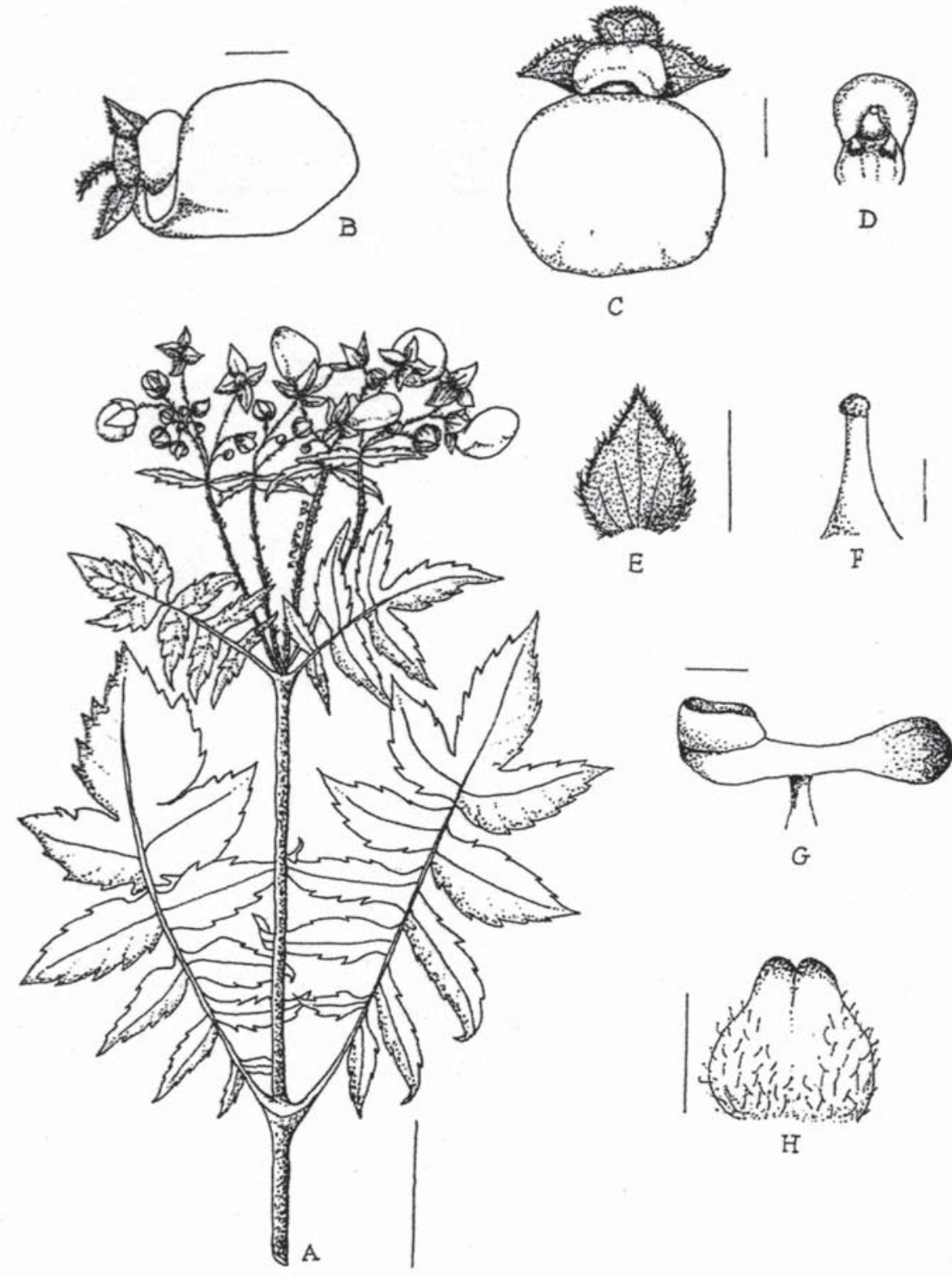

$\mathrm{H}$

Figura 1. Calceolaria chelidonioides Kunth. A. Rama; B. Flor, vista lateral; C. Flor, vista frontal, D. Corola, labio superior; E. Sépalo, vista ventral; F. Estilo; G. Estambre, vista lateral; H. Cápsula, vista frontal. Escalas: A. 5 cm; B, C, D, E, H, 5 mm; F, G, 1 mm (dibujo tomado de Puppo 2005).

Figure 1. Calceolaria chelidonioides Kunth. A. Branch; B. Flower, lateral view; C. Flower, frontal view; D. Corolla, upper lip; E. Sepal, ventral view; F. Style; G. Stamen, lateral view; H. Capsule, frontal view. Scales: A. 5 cm; B, C, D, E, H, 5 mm; F, G, 1 mm (drawing taken from Puppo 2005). 
Distribución: Crece desde Colombia a Chile por ambas vertientes de la cordillera de los Andes. En Chile esta especie ha sido colectada en su mayoría en la Región de Valparaíso, confinada a una extensión de $140 \mathrm{~km}$ de latitud (Zapallar a San Antonio). En cuanto a su distribución altitudinal en estas regiones, ha sido hallada ente los 5-900 m sobre el nivel del mar.

Material Revisado: Región de Valparaíso, 1838-1842. Wilkes Explor. Exped. s.n. (US 960822); X-1883. Curauma s.n. (SGO); Zapallar, 05-I-1917. Anónimo s.n. (F 685098); Zapallar, Quebrada del Infierno, $32^{\circ} 33^{\prime} \mathrm{S}, 71^{\circ} 28^{\prime} \mathrm{O}, 50 \mathrm{~m}$. 05-I-1917. K. Behn s.n. (CONC); Zapallar, Quebrada del

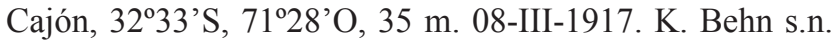
(CONC); Camino a Concón, 3255'S, 7130'O, 15 m. 25IX-1927. K. Behn s.n. (CONC); Viña del Mar, 21-IX-1930.

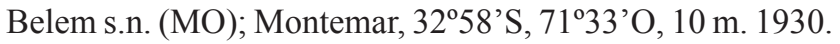
Garaventa 1574 (CONC); Quintero, 32 ${ }^{\circ} 47^{\prime} \mathrm{S}, 71^{\circ} 32^{\prime} \mathrm{O}, 40$ m. 23-III-1940. Looser 4719 (CONC); Quintero, 3247'S, 71³2'O, 40 m. 1950. Richter s.n. (CONC); Bosque Los Arrayanes, 32²46'S, 7132'O, $20 \mathrm{~m}$. XI-1952. Gunckel 48561

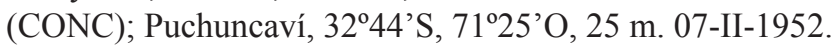
Levi 346 (CONC); Horcón, 32²47'S, 71³2'O, 10 m. 12-II1953. Gunckel 23504 (CONC); Concón, $32^{\circ} 55^{\prime} \mathrm{S}, 71^{\circ} 31^{\prime} \mathrm{O}$, 25 m. 20-II-1953. Gunckel 24079 (CONC); Papudo, 100 m. 01-XI-1960. M.L. Véliz C. s.n. (SGO); Concón, en agua al lado del camino, 5 m. 07-X-1973. G.L. Stebbins 8599 (SGO); Estero Aguas Claras, entre Maitencillo y Cachagua, 04-XI-1988. P. Riedemann s.n. (SGO); Dunas de Concón 32 $55^{\circ} 46^{\prime}$ "S, 7132'53”'O, 5 m. 07-X-2002. A. Moreira 675 C (SGO); Cuming 615 (CONC); Camino Costero ReñacaConcón, 3259'07,92”S, 71³0’08,17’O. 28-XI-2012. P. Novoa 175177 (CONC); Cerro Tres Puntas, $33^{\circ} 05^{\prime} \mathrm{S}$,

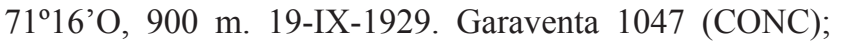
Rocas de Santo Domingo, las vertientes, $33^{\circ} 38^{\prime} \mathrm{S}, 71^{\circ} 38^{\prime} \mathrm{O}$, 70 m. 22-II-1965, Ricardi 5245 (CONC).

2. Calceolaria pinnata L. subsp. pinnata, Kongl. Vetensk. Acad. Handl. 31: 286. 1770. Fagelia flavicans Gmelin, Syst. 2: 40. 1791. Calceolaria pulchella Salisbury, Podr. Stirp. Chap. Allerton p. 89. 1796. TIPO: Suecia. Cultivada en Uppsala, propagada de semillas recibidas de Jussieu, colectadas en vic. Lima, Perú. Linnaeus s.n. (lectotipo LINN, no visto). Fig. 2.

Calceolaria bipinnatifida Phil., Fl. Atacam. p. 46. 1860. TIPO: Chile. Antofagasta: Miguel Díaz (Punta Dos Reyes), nr. the Pacific coast. XII-1853. Philippi s.n. (lectotipo SGO; isotipos $\mathrm{L}, \mathrm{W}, \mathrm{ZT}$ ).

Hierba de hasta $60 \mathrm{~cm}$ de alto, tallos glabrescentes o glandular-villosos en el extremo distal, verdes. Hojas pinnatisectas $3-11 \times 2-7,5 \mathrm{~cm}$, hasta 3 pares de pinnas laterales, pinnas ovadas, márgenes profundamente lobados; peciolos 1,5-3,5 cm long. con un pequeño ensanchamiento en la base de hasta $4 \mathrm{~mm}$ y frecuentemente connados al tallo. Sépalos ovados, 4-6 x 2,5-4 mm, verdes, agudos, margen entero, externamente pilosos. Corola 10-25 mm long., amarilla, glabra o con pequeñas glándulas. Estambres con tecas dimorfas, la posterior de 1,5-2 mm long., la anterior de 0,9-1,1 mm long., conectivo 1-1,5 mm long., sigmoideo. Cápsula ovada 5-7 mm long., glandular cerca a la base.

Distribución: Esta subespecie es nativa de Perú y Chile. En Chile es conocida únicamente en las regiones de Atacama y Antofagasta. No se tienen datos sobre su distribución altitudinal en Chile, pero se sabe que esta especie cuando presente en formaciones de lomas costeras habita entre los 200-900 m sobre el nivel del mar (Molau 1988).

Comentarios: Molau (1988, p. 247) indica que tanto Fagelia flavicans como Calceolaria pulchella son sinónimos obligatorios de C. pinnata. En el caso de C. pulchella, nunca hubo cambio de nombre ya que la designación de "pulchella" fue únicamente usada por Salisbury para describir C. pinnata L. y no para designar una nueva especie (ver Salisbury 1796, p. 89). En el caso de F. flavicans, Gmelin cita la publicación donde se designa el género Fagelia Schwencke (ver Gmelin 1791 v.2, p. 40). Al parecer, Schwencke habría designado $F$. flavicans como tipo de este nuevo género. Lamentablemente, no hay más datos sobre la publicación de Schwencke ya que no aparece ni en IPNI (2008) ni en Botanicus.org.

Material Revisado: Región de Antofagasta, Miguel Díaz (Punta de los Reyes), nr the Pacific Coast, XII-1932. Philippi s.n. (SGO).

3. Calceolaria tripartita Ruiz \& Pav., Fl. Peruv. 1: 14. 1798. Fagelia tripartita (Ruiz \& Pav.) Kuntze, Revis. Gen. Pl. 2: 460. 1891. TIPO: Perú. "In nemoribus Panatahuas". Pavón s.n. (TIPO: perdido, ver Pennell 1945, p.174). NEOTIPO: Perú. Lima: Prov. Canta, a lo largo del Río Chillón, sobre Obrajillo, NE de Canta, 3100-3300 m. 13-23-VI-1925. Pennell 14406 (neotipo PH, isoneotipos F!, G!, LE, NY!, US, designado por Molau 1981, p.605). Fig. 3.

Calceolaria heterophylla Willdenow, Enum. Pl. 1:29. 1809. Calceolaria scabiosaefolia Roemer \& Schultes, Syst. Veg. ed. 15, 1: 187. 1817. TIPO: Perú. Huánuco: Prov. Huánuco, Huánuco, Pavón s.n. (lectotipo MA; isotipos FI, G, MPU).

C. gracilis Kunth, Nov. Gen. Sp. Pl. 2: 379. 1818. Fagelia gracilis (Kunth) Kuntze, Rev. Gen. P1. 2: 459. 1891. TIPO: Ecuador. Pichincha: Río San Pedro, sobre Chillo, SE Quito, ca. $2450 \mathrm{~m}$. VI-1802. Bonpland 3109 (lectotipo B-WILD; isotipos $\mathrm{P}, \mathrm{W}$ ). 


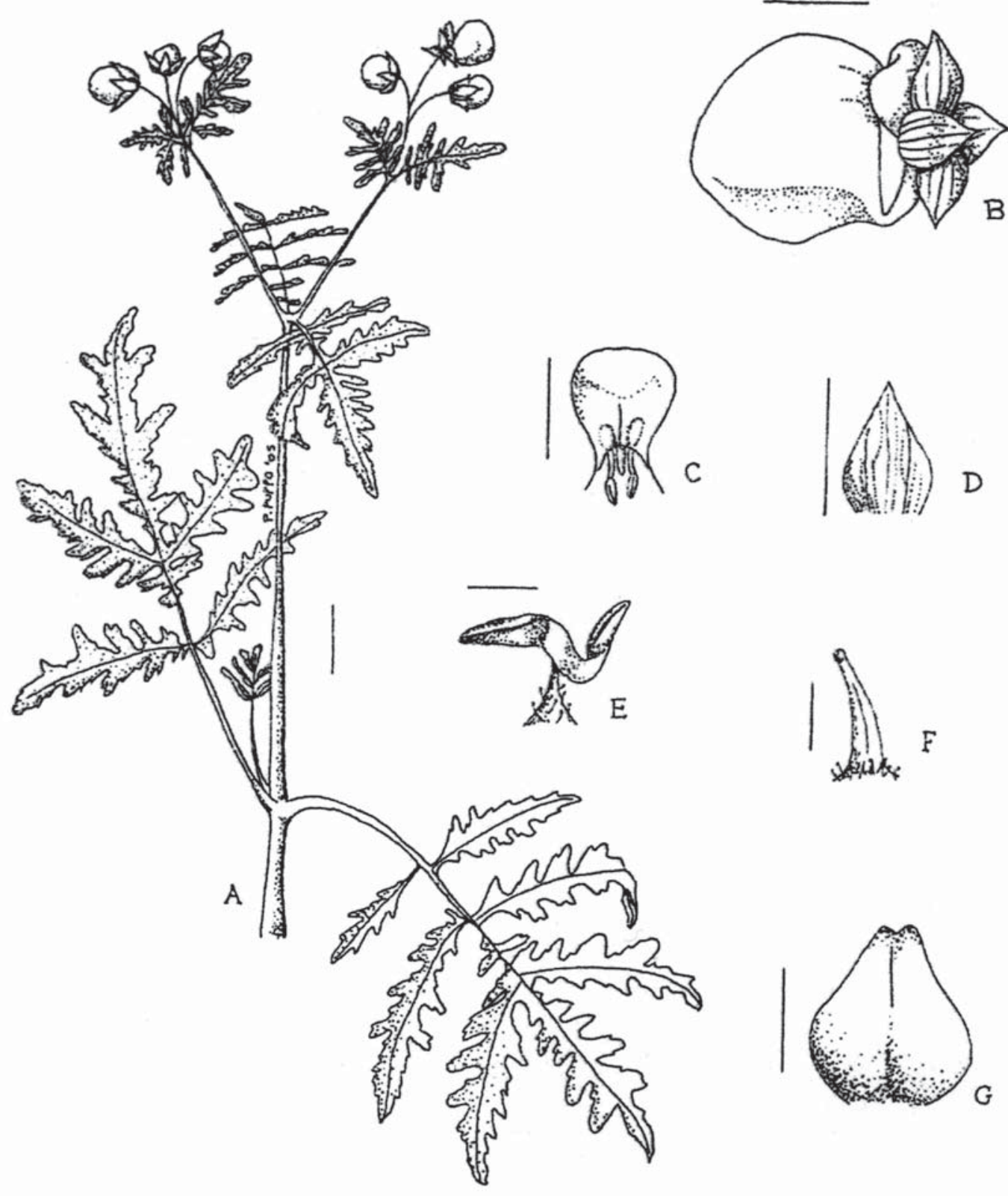

Figura 2. Calceolaria pinnata L. A. Rama; B. Flor, vista lateral; C. Corola, labio superior; D. Sépalo, vista ventral; E. Estambre, vista lateral; F. Estilo; G. Cápsula, vista frontal. Escalas: A. 1 cm; B, C, D, G, 5 mm; E, F, 1 mm (dibujo tomado de Puppo 2005).

Figure 2. Calceolaria pinnata L. A. Branch; B. Flower, lateral view; C. Corolla, upper lip; D. Sepal, ventral view; E. Stamen, lateral view; F. Style; G. Capsule, frontal view. Scales: A. 1 cm; B, C, D, G, 5 mm; E, F, 1 mm (drawing taken from Puppo 2005). 


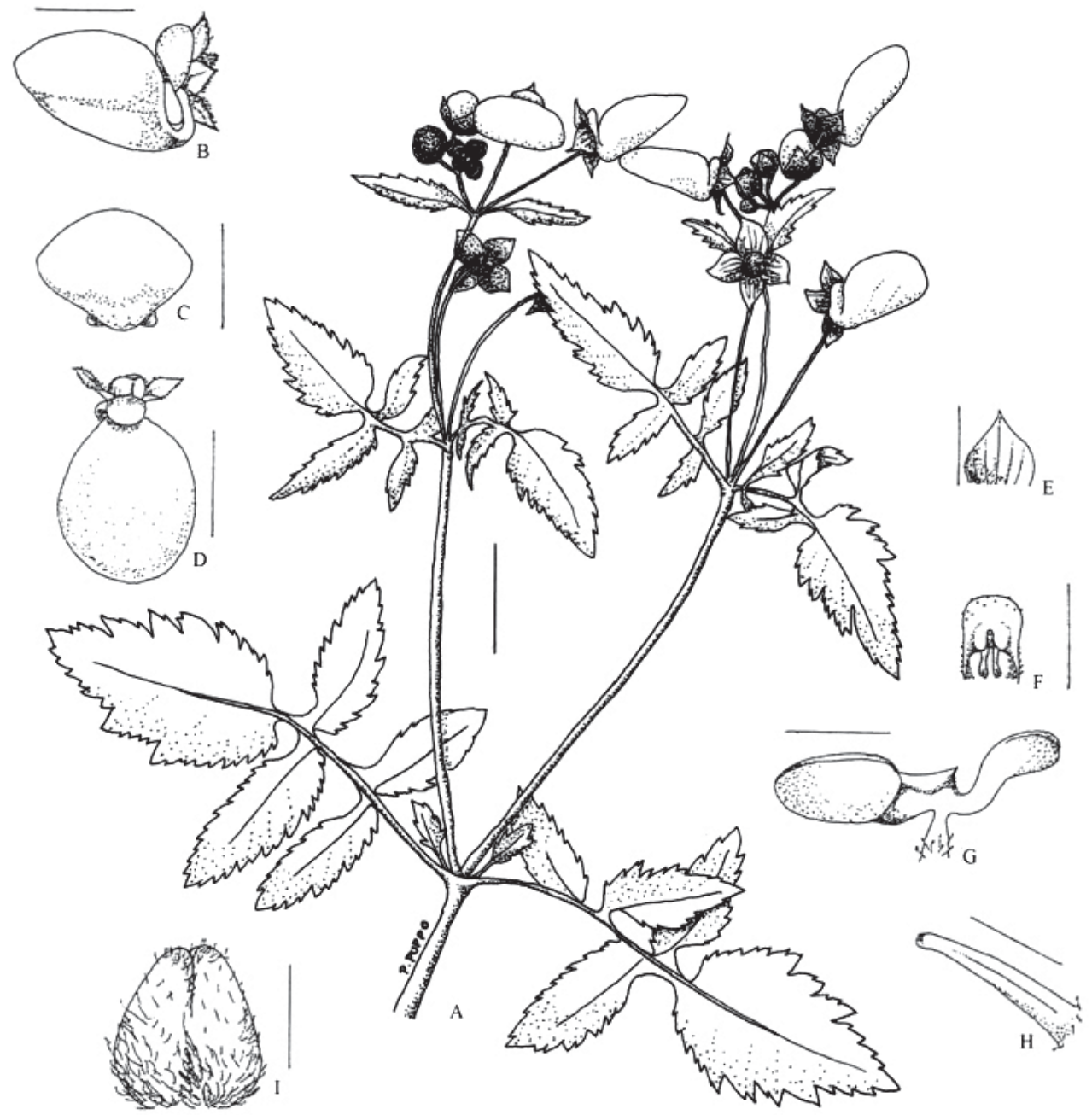

Figura 3. Calceolaria tripartita Ruiz \& Pav. A. Rama; B. Flor, vista lateral; C. Flor, vista frontal; D. Flor, vista superior; E. Sépalo, vista ventral; F. Corola, labio superior; G. Estambre, vista lateral; H. Estilo; I. Cápsula, vista frontal. Escalas: A. 2 cm; B, C, D, F, 1 cm; E, I, 5 mm; G, H, 2 mm (A. tomado de Puppo 2008; B.-I. tomado de Puppo 2005).

Figure 3. Calceolaria tripartita Ruiz \& Pav. A. Branch; B. Flower, lateral view; C. Flower, frontal view; D. Flower, upper view; E. Sepal, ventral view; F. Corolla, upper lip; G. Stamen, lateral view; H. Style; I. Capsule, frontal view. Scales: A. 2 cm; B, C, D, F, 1 cm; E, I, 5 mm; G., H., 2 mm (A. was taken from Puppo 2008; B.-I. from Puppo 2005).

C. chelidonioides Kunth $\beta$ parvifolia Benth., in DC., Prodr. 10: 204. 1846. TIPO: Ecuador. Pichincha: Rumipampa, SE Quito, Hartweg 1271 (lectotipo K, isotipos BM, BREM, CGE, E, FI, G, LD, LE, NY, OXF, P, W).

C. chelidonioides $\gamma$ flaccida Benth., in DC., Prodr. 10:204. 1846. TIPO: Ecuador. Pichincha: Quito, Hall 3 (lectotipo K).

C. ranunculoides Kraenzlin, Feddes Repert. Spec. Nov. Regni Veg. 1: 97. 1905. TIPO: Perú. Ancash: Prov. Bolognesi, Ocros, 3500 m. Weberbauer 2708 (holotipo B [destruido], F [foto]; lectotipo G; isotipos MOL).
C. sarmentosa Kraenzlin, Feddes Repert. Spec. Nov. Regni Veg. 1: 97. 1905. TIPO: Perú. Ancash: Prov. Huaylas, entre Caraz y Samando, $3100 \mathrm{~m}$. Weberbauer 3121 (holotipo B [destruido], F [foto]; lectotipo G; isotipos MOL).

Fagelia scalaris Pennell, Proc. Acad. Nat. Sci. Philadelphia 72: 184. 1920. TIPO: Colombia. Caquetá: Hda. Balsillas en el río Balsillas, laderas E de la Cordillera Oriental, E de Neiva, 2000-2100 m. 3-6-VIII-1917. Rusby \& Pennell 710 (holotipo NY; isotipos K, NY, US).

C. puru-puru Kraenzlin, Feddes Repert. Spec. Nov. Regni Veg. 27: 2. 1929. TIPO: Perú. Cuzco: Prov. Urubamba, 
Yucay, SE de Urubamba, 2900 m. VIII-1925. Herrera 748 (holotipo B [destruido]; lectotipo F [foto]).

C. micrantha Pennell, Proc. Acad. Nat. Sci. Philadelphia 97: 176. 1945. TIPO: Perú. Cuzco: Prov. Paucartambo, cerca al rio Yanamayo debajo de Pillahuata, 2200-2400 m. 4-5-V1925. Pennell 14041 (holotipo PH; isotipos CUZ, F, GH, K, NY, US).

C. chelidonioides subsp. serratifolia López Guillén, Raymondiana 1: 59. 1968. TIPO: Perú. Cajamarca: Prov. Cajamarca. 2-II-1967. López Guillén 3432 (holotipo SMF).

C. soukupii López Guillén, Raymondiana 1:34. 1968. TIPO: Perú. La Libertad: Prov. Sánchez Carrión, a lo largo del rio Colorado. 22-II-1967. López Guillén 3528 (holotipo SMF).

C. tripartita subsp. cajamarcensis López Guillén, Raymondiana 1: 49. 1968. TIPO: Perú. Cajamarca: Prov. Cajamarca, Llacanora. 4-II-1967. López Guillén 3430 (holotipo SMF).

C. tripartita subsp. candelabrensis López Guillén, Raymondiana 1: 53. 1968. TIPO: Perú. Sine loco. López Guillén 1740 (holotipo SMF).

C. tripartita subsp. chumpitazii López Guillén, Raymondiana 1: 50. 1968. TIPO: Perú. Cajamarca: Prov. Cajamarca, Llacanora. 4-II-1967. López Guillén 3423 (holotipo SMF).

C. tripartita subsp. crenatifolia López Guillén, Raymondiana 1: 55. 1968. TIPO: Perú. Sine loco. López Guillén 1743 (holotipo SMF).

C. tripartita subsp. diversifolia López Guillén, Raymondiana 1: 55. 1968. TIPO: Perú. Sine loco. López Guillén 1477 (holotipo SMF).

C. tripartita subsp. inaequalis López Guillén, Raymondiana 1: 52. 1968. TIPO: Perú. Sine loco. López Guillén 1559 (holotipo SMF).

C. tripartita subsp. longipedunculata López Guillén, Raymondiana 1: 54. 1968. TIPO: Perú. Sine loco. López Guillén 3423 (holotipo SMF).

C. tripartita subsp. retroflexa López Guillén, Raymondiana 1: 51. 1968. TIPO: Perú. Sine loco. López Guillén 1274 (holotipo SMF).

C. velardei López Guillén, Raymondiana 1: 41. 1968. TIPO: Perú. Huánuco: Prov. Huánuco, vic. Huánuco, 3245 m. 26IV-1957. Infantes 1273 (holotipo SMF).
C. celendinensis López Guillén, Raymondiana 2: 16. 1969. TIPO: Perú. Cajamarca: Prov. Celendín, montañas de Jelic. 14-III-1969. Riccio 6279 (holotipo SMF).

C. chelidonioides subsp. truncata López Guillén, Raymondiana 2: 20. 1969. TIPO: Perú. Cajamarca: Prov. Celendín, cerro La Soma, camino a Rumi Rumi, distrito de Sucre. 13-V-1969. Riccio 6343 (holotipo SMF).

C. corazonica Gilli, Feddes Repert. Spec. Nov. Regni Veg. 94: 318. 1981. TIPO: Ecuador. Cotopaxi: laderas O de la Cordillera Occidental, cerca a El Corazón, 1440 m. 28-VI1975. Gilli 226 (holotipo W).

Hierba de hasta $1 \mathrm{~m}$ de alto, tallos superiores glandularvillosos, verde. Hojas pinnatisectas $(0,8) 2-14$ x $(0,7) 2-16$ $\mathrm{cm}$, hasta 4 pares de pinnas laterales, pinnas ovadas, agudas al ápice, márgenes frecuentemente crenados o serrados; en especímenes pequeños o poco desarrollados las hojas pueden parecer enteras a pinnatipartidas; peciolos $1-6 \mathrm{~cm}$ long. con un ligero ensanchamiento en la base alcanzando hasta $6 \mathrm{~mm}$. Sépalos ovados, 2-10 x 1-7 mm, verdes, agudos, margen entero o dentado, externamente glandular-pilosos. Corola 6-29 mm long., amarilla, glabra o con pequeñas glándulas. Estambres con una sola teca fértil (la posterior) de 0,7-2,5 $\mathrm{mm}$ de long., la teca anterior abortada, conectivo alargado 0,8-4,1 mm long., sigmoideo hacia el extremo anterior y con una protuberancia dorsal. Cápsula ovada o subglobosa 5-10 mm long., glandular estrigosa.

Distribución: Habita en América Latina desde el sur de México hasta Bolivia. Ha sido encontrada también en algunas islas del Océano Pacífico, Océano Atlántico y en varios países del Continente Asiático (ver Molau 1981, 1988). En Chile, C. tripartita ha sido colectada únicamente en la provincia de Valparaíso entre los $15-70 \mathrm{~m}$ sobre el nivel del mar.

MATERIAL ReVISADO: Región de Valparaíso, Zapallar, 32³3'S,

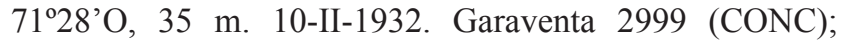

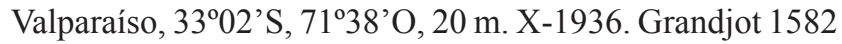
(CONC); Camino Viña del Mar a Concón, 15 m. 27-IX-1945. Pfister s.n. (CONC); Limachito, Estero Pelamote, $33^{\circ} 01^{\prime}$ S, 71 ${ }^{\circ} 16^{\prime} \mathrm{O}, 70$ m. 12-XI-1950. Garaventa 5352 (CONC); Quintero, Los Arrayanes, 3246'S, 7132'O, 20 m. XI-1952. Gunckel 23800 (CONC); Quintero, Los Arrayanes, $32^{\circ} 47^{\prime} \mathrm{S}$, 713'ㅇ, 40 m. XI-1952. Navas 1329 (CONC); Bosque Hacienda Pullalli, I-1970. P. Dreckmann s.n. (SGO); Viña del Mar, Cochoa, frente a Puerto Maldonado, 11-XI-2002. M. Chamy \& M. Piovano 31 (SGO); Quillota, Ph. Germain s.n. (SGO 055907); Quillota, Ph. Germain s.n. (PH 9858); Anónimo s.n. (SGO 042986). Sine loco, Anónimo s.n. (NY); Anónimo s.n. (PH 01068689). 


\section{DISCUSIÓN}

En Chile existen 50 especies de Calceolaria según la última revisión del género hecha para este país (Ehrhart 2000). Estas especies se han agrupado en 4 secciones: Calceolaria, Cheiloncos, Kremastocheilos y Tenelle (Ehrhart 2000).

Las especies de la sección Calceolaria que crecen en Chile están ampliamente distribuidas en el Neotrópico y constituyen especies características de zonas con abundante agua creciendo frecuentemente en las márgenes de arroyos o riachuelos. A pesar de ser tan comunes en estas áreas, en Chile sólo se conocían 2 colecciones de una especie de esta sección, C. pinnata subsp. pinnata: Johnston 5420 y Philippi s.n. (citadas por Molau 1988 y Ehrhart 2000) recolectadas en la Región de Antofagasta. En el presente trabajo se dan a conocer más de 35 especímenes de 3 especies depositados mayoritariamente en los herbarios chilenos CONC y SGO.

En la presente revisión no se encontraron especímenes adicionales de $C$. pinnata subsp. pinnata además de los dos ya mencionados. La situación de esta especie es preocupante ya que estas colecciones son de 1853 y 1925. ¿Será posible que $C$. pinnata haya desaparecido del territorio chileno? o ila falta de material en los herbarios se deba a falta de colecciones? Molau (1988, p. 248) hablando de las poblaciones de esta especie en Perú sugería que se tratase como un taxón amenazado por la rápida destrucción de las lomas costeras, principal hábitat de esta especie.

Más de la mitad del material que encontramos en los herbarios chilenos estaban determinados como $C$. scabiosifolia. Sin embargo, existen tres publicaciones con especies descritas bajo este nombre (ver IPNI 2008).

La primera es C. scabiosifolia Roem. \& Schult. publicada originalmente como C. sabiosaefolia en 1817 (ver Roemer \& Schultes 1817, p.187). Esta especie fue descrita para enmendar la especie publicada por Willdenow en 1809, C. heterophylla. Willdenow habría descrito esta especie usando un epíteto específico ya usado por Ruiz \& Pavón en 1798. Algunos años más tarde, en 1822, Schultes hace una revisión de su publicación de 1817 en donde declara que el nombre de C. scabiosifolia debería ser "borrado" ya que se trata de $C$. gracilis Kunth publicada también en 1817 (ver Schultes 1822, [97]163). En revisiones más recientes, C. gracilis es considerada sinónimo de C. tripartita (ver por ejemplo: Pennell 1951, Molau 1981, 1988).

La segunda C. scabiosifolia es la publicada por Sims en 1823 (citada por Gay 1845, Reiche 1911 y Johow 1948). Esta especie fue publicada de un material propagado de semillas provenientes de Chile (Sims 1823, No 2405). Sims incluye una ilustración detallada de la especie, pero no designa un tipo para la misma ni da indicación de dónde fue depositado el espécimen en el cual basó su descripción. Además indica como sinónimos de esta especie a C. scabiosifolia Roem. \& Schult., C. heterophylla Willd., y C. pinnata Ruiz \& Pav., estas tres consideradas como $C$. tripartita en la revisión más reciente del género (Molau 1988). Es de resaltar que $C$. scabiosifolia Sims no se encuentra citada en la publicación de Molau (1988). Únicamente se encuentra una referencia a una C. sabiosifolia var. cicutifolia descrita por Kraenzlin en 1907 y que Molau cita como sinónimo de C. tenuis, otra especie de la sección Calceolaria que presenta ambas tecas fértiles (ver Molau 1988, p. 249, Kraenzlin 1907, p. 23-24). Al consultar la ilustración que acompaña la especie descrita por Sims vemos claramente que se trata de $C$. chelidonioides debido a la forma pinnatisecta de las hojas, las bases de los peciolos ensanchados y connados al tallo, los estambres con sólo una teca fértil y el conectivo prolongado, casi recto y sin una protuberancia dorsal. Por otro lado, sería interesante saber cuál era la idea que Reiche tenía de $C$. chelidonioides ya que él menciona en su publicación de 1911 que "en los últimos 80 años no se ha visto por nadie en Chile" cuando él sí la había visto pero la llamaba C. scabiosifolia Sims.

La tercera C. sabiosifolia conocida es C. sabiosaefolia Nees ex G. Don. Sin embargo, la única referencia que se tiene de esta especie es en la publicación de Don (1838), donde es citada apenas como sinónimo de C. rugosa Ruiz \& Pav., la cual pertenece a otra sección del género (sect. Cheiloncos sensu Ehrhart 2000, sect. Rugosae sensu Molau 1988).

En el presente trabajo se encontraron más de 20 ejemplares de $C$. chelidonioides, la mayoría colectados hace más de 60 años. Sin embargo, sabemos que la especie continúa creciendo en Chile ya que investigadores del Jardín Botánico de Viña del Mar han encontrado recientemente poblaciones de esta especie creciendo en afloramientos de agua en el camino costero que une las ciudades de Viña del Mar y Concón en la Región de Valparaíso, en la quebrada Rodelillos de Viña del Mar y en la quebrada El Boldo del pueblo de Zapallar ubicado a $56 \mathrm{~km}$ al norte de Viña del Mar. Calceolaria chelidonioides ha sido considerada sinónimo de C. tripartita en algunas revisiones taxonómicas anteriores (Edwin 1971, Molau 1979). Ambas son de distribución bastante amplia y hay áreas en donde ocurren en simpatría. Sin embargo, según la última revisión del género hecha por Molau (1988) se trata de dos especies diferentes y hasta que no haya un estudio pormenorizado que pruebe si se trata de una especie o dos, es mejor tratarlas por separado.

Ehrhart (2000) hace un comentario al final de su cita de C. pinnata sobre otra especie de la sección Calceolaria presente en Chile: $C$. tripartita. Según Ehrhart, esta especie ha sido evidentemente introducida en Valparaíso. Más aún, ella afirma que algunas colecciones son conocidas de la primera mitad del siglo 20, pero que al tratarse de una especie no nativa de Chile no es discutida en detalle en su monografía (Ehrhart 2000, p. 85). Podemos concluir pues que Ehrhart tenía conocimiento de la presencia de $C$. tripartita en Chile sin embargo no le pareció importante incluirla en su trabajo. En la presente revisión encontramos más de 10 ejemplares de C. tripartita, aunque es cierto 
que la mayoría de éstos fueron recolectados en la primera mitad del siglo pasado, hay una colecta del 2002 (Chamy \& Piovano 31) que nos indica que esta especie continúa presente en Valparaíso. Las localidades indicadas para esta especie incluyen las quebradas en Zapallar y en La Vega, los arrayanes en Quintero, Concón y Limachito. Aunque Ehrhart (2000) la trate como introducida, esta aseveración debe tomarse con cautela ya que las calceolarias de agua fueron citadas para los mismos lugares donde hoy crecen desde la indicación de Poeppig (citada por Reiche), quien visitó Valparaíso y Concón en 1827, y los trabajos de Gay en 1845, desde esas fechas han permanecido en la misma zona original, la costa de la Región de Valparaíso, sin mostrar expansión o comportamiento de maleza.

Se encontró también un ejemplar chileno de Poeppig s.n. sin localidad depositada en $\mathrm{PH}$, la cual aparece como C. rivularis Kraenzl. (sección Calceolaria sensu Molau 1988) en la base de datos de este herbario (PH 9499). Sin embargo, al examinar la foto en alta resolución del ejemplar verificamos que se trataba de C. petiolaris Cav., especie perteneciente a la sección Cheiloncos (sensu Ehrhart 2000). Al igual que las especies de la sección Calceolaria, $C$. petiolaris habita en lugares húmedos, aunque esta especie es fácilmente reconocida por su hábito y morfología estaminal. Calceolaria petiolaris presenta las hojas basales formando una roseta, inflorescencias laxas con brácteas oblongas, sésiles y connadas a los tallos, y filamentos significativamente más largos que las anteras.

\section{ESTADO DE CONSERVACIÓN}

Calceolaria pinnata ssp. pinnata esta probablemente Extinta en Estado Silvestre (EW) en Chile ya que no ha sido recolectada desde inicios del siglo XX.

\section{AGRADECIMIENTOS}

En primer lugar nos gustaría agradecer a los curadores de los siguientes herbarios: CONC, F, MO, NY, PH, SGO, US. Un reconocimiento especial a Marcelo Baeza por fotografiar el material depositado en el herbario CONC, a Alina FreireFierro por fotografiar dos ejemplares depositados en $\mathrm{PH}$, a Jorge Macaya por comentarios durante la preparación del documento y a los dos revisores anónimos que ayudaron con sus sugerencias a mejorar el manuscrito.

\section{BIBLIOGRAFÍA}

Andersson, S. 2006. On the phylogeny of the genus Calceolaria (Calceolariaceae) as inferred from ITS and plastid matK sequences. Taxon 55: 125-137.

Cosacov, A., A.N. Sérsic, V. Sosa, J.A. De-Nova, S. Nylinder \& A.A. Cocucci. 2009. New insights into the phylogenetic relationships, character evolution, and phytogeographic patterns of Calceolaria (Calceolariaceae). American Journal of Botany 96: 2240-2255.

Don, G. 1838. A General History of the Dichlamydeous Plants. London. v. 4.908 pp.

EDwIN, G. 1971. Scrophulariaceae. Flora of Peru. Botanical Series, Field Museum of Natural History 13(5-B): 461-717.

Ehrhart, C. 2000. Die Gattung Calceolaria (Scrophulariaceae) in Chile. Bibliotheca Botanica 153: 1-283.

GAY, C. 1845. Historia física y política de Chile. Botánica (Flora de Chile). Tomo V.

Gmelin, J.F. 1791. Caroli a Linné...Systema naturae per Regna Tria Naturae. Lipsiae. v.2. 884 pp.

International Plant Names Index (IPNI). 2008. Published on the Internet http://www.ipni.org [Accesado 29 marzo 2012].

JoHow, F. 1948. Flora de las plantas vasculares de Zapallar. Revista Chilena de Historia Natural 49: 1-566.

Kraenzlin, F. 1907. Scrophulariaceae-AntirrhinoideaeCalceolarieae. En: A. Engler, Das Pflanzenreich v. 4 (257C): 1-128.

Marticorena, C. \& M. Quezada. 1985. Catálogo de la Flora Vascular de Chile. Gayana Botánica 42: 1-155.

Molau, U. 1979. Calceolaria. In: D’Arcy, Flora of Panama, Family 171. Scrophulariaceae. Annals of the Missouri Botanical Garden 66: 202-208.

Molau, U. 1981. The genus Calceolaria in NW South America. VIII. The section Calceolaria and appendices to parts IVIII. Nordic Journal of Botany 1: 595-615.

Molau, U. 1988. Scrophulariaceae. Part I. Calceolarieae. Flora Neotropica Monographs 47: 1-326.

Olmstead, R. \& P.A. ReEves. 1995. Evidence for the polyphyly of the Scrophulariaceae based on chloroplast $r b c l$ and $n d h F$ sequences. Annals of the Missouri Botanical Garden 82: 176-193.

Olmstead, R., C. de Pamphilis, A. Wolfe, N. Young, W. Elisons \& P. Reeves. 2001. Desintegration of the Scrophulariaceae. American Journal of Botany 88: 348-361.

Pennell, F.W. 1951. The genus Calceolaria in Ecuador, Colombia and Venezuela. Proceedings of the Academy of Natural Sciences of Philadelphia 103: 85-196.

Puppo, P. 2005. Estudio Taxonómico del género Calceolaria L. en la cuenca alta y media del Río Chillón -Provincia de Canta. Tesis para optar al título de Biólogo. Facultad de Ciencias, Universidad Nacional Agraria La Molina, Lima, Perú. 108 pp.

Puppo, P. 2006. El género Calceolaria (Calceolariaceae) en el departamento de Lima-Perú. Revista Peruana de Biología 13: 85-93.

Puppo, P. 2008. Taxonomic revision of the Calceolaria tripartita complex (Calceolariaceae). Masters of Science thesis, University of Missouri St. Louis, U.S.A. 76 pp.

Reiche, K.F. 1911. Flora de Chile. Santiago de Chile. v.6. 178 pp.

Roemer, J.J. \& J.A. Schultes. 1817. Caroli a Linné...Systema vegetabilium: secundum clases, ordines, genera, species. Editio nova, speciebus inde ab editione XV. Detectis aucta et locupletata. Stuttgardtiae. v. 1. 642 pp.

Ruiz, H. \& J. PAvón. 1798. Flora Peruviana et Chilensis. Madrid. v. $1.78 \mathrm{pp}$.

Salisbury, R.A. 1796. Prodromus Stirpium in Horto ad Chapel Allerton Vigentium. London. $422 \mathrm{pp}$.

Schultes, J.A. 1822. Mantissa in Volumen Primum. Systematis 
Vegetabilium Caroli a Linné. Stuttgardtiae. 386 pp.

Sims, J. 1823. Curtis's Botanical Magazine. London. v. 50. 237 pp.

Thiers, B. 2011. Index Herbariorum: A global directory of public herbaria and associated staff. New York Botanical Garden's Virtual Herbarium. URL: http://sweetgum.nybg.org/ih/ [Accesado 01 agosto 2011].

UICN. 2001. Categorías y Criterios de la Lista Roja de la UICN: Versión 3.1. Comisión de Supervivencia de Especies de la UICN. UICN, Gland, Suiza y Cambridge, Reino Unido. ii $+33 \mathrm{pp}$.
Villagrán, C., C. Marticorena \& J.J. Armesto (eds.). 2007. Flora de las plantas vasculares de Zapallar. Revisión ampliada e ilustrada de la obra de Federico Johow. Ed. Universidad Metropolitana de Ciencias de la Educación. Santiago. 646 pp.

Vogel, S. 1974. Ölblumen und ölsammelnde Bienen. Zweite Folge. Tropische und Subtropische Pflanzenwelt 7: 1-276.

Willdenow, C.L. 1809. Enumeratio Plantarum Horti Regii Botanici Berolinensis. Berolini. 1099 pp.

Recibido: 09.12.11

Aceptado: 20.04.12 